\title{
An Information-theoretic Approach to Network Monitoring and Measurement
}

\author{
Yong Liu \\ ECE Dept. \\ Polytechnic University \\ Brooklyn, NY 11201
}

\author{
Don Towsley \\ Dept. of Computer Science \\ University of Massachussetts \\ Amherst, MA 01003 \\ Jean Bolot \\ Sprint ATL \\ Burlingame, CA 94010
}

\author{
Tao Ye \\ Sprint ATL \\ Burlingame, CA 94010
}

\begin{abstract}
Network engineers and operators are faced with a number of challenges that arise in the context of network monitoring and measurement. These include: i) how much information is included in measurement traces and by how much can we compress those traces?, ii) how much information is captured by different monitoring paradigms and tools ranging from full packet header captures to flow-level captures (such as with NetFlow) to packet and byte counts (such as with SNMP)? and iii) how much joint information is included in traces collected at different points and can we take advantage of this joint information? In this paper we develop a network model and an information theoretic framework within which to address these questions. We use the model and the framework to first determine the benefits of compressing traces captured at a single monitoring point, and we outline approaches to achieve those benefits. We next consider the benefits of joint coding, or equivalently of joint compression of traces captured a different monitoring points. Finally, we examine the difference in information content when measurements are made at either the flow level or the packet/byte count level. In all of these cases, the effect of temporal and spatial correlation on the answers to the above questions is examined. Both our model and its predictions are validated against measurements taken from a large operational network.
\end{abstract}

\section{Introduction}

Network monitoring is an immense undertaking in a large network. It consists of monitoring (or sensing) a network using a geographically distributed set of monitoring stations, with the purpose of using the monitoring data to better understand the behavior of the network and its users. Monitoring is a central activity in the design, engineering, and operation of a network. Increased mon- itoring capabilities, along with the associated increased understanding of the network and user behavior (or misbehavior), have direct impact on network performance and integrity, and therefore on the costs passed on to network users, and on the revenues of the operators. In spite of its importance, practitioners struggle with challenging, yet very practical questions such as where within the network to monitor data and at what granularity to capture traces, how much information is included in various types of packet traces and by how much can we compress those traces, and how much joint information is included in traces collected at different points and how can we take advantage of this joint information?

In this paper, we address these questions in the context of large high speed networks such as campus, enterprise, or core networks. Monitoring the behavior of such networks raises tremendous challenges due to the high bandwidth of currently deployed links. For example, the collection of 60-byte packet headers can easily generate $3 \mathrm{~Tb}$ of data per hour on a OC-192 link (10 Gb/s link) in a core backbone, and $30 \mathrm{~Gb}$ of data per hour at an enterprise or campus gateway. One means for reducing the amount of data gathered is to monitor flow-level data, as is done with Net Flow [2]. The amount of data can be further reduced by monitoring packet or byte counts over fixed intervals of time as is possible using SNMP [15]. Network data collected at distributed monitors also exhibit spatial and temporal correlations. Thus another means for reducing the sizes of monitored data sets is to exploit this correlation through correlated data coding and compression.

In this paper, we propose an information theoretic framework within which to address some of the issues and questions introduced above. In particular, we propose and validate a flow-level model (adapted from [12]), and we use it to determine the information content of a packet trace collected at a single or multiple points in a network, and of sets of packet traces collected at separate points. We also determine the information content of 
traces captured at different levels of granularity, in particular flow level traces (NetFlow traces) and byte or packet count traces (SNMP traces).

We obtain a number of interesting and important results. Regarding traces collected at a single monitoring point, we derive an information theoretic bound for the information content in those traces, or equivalently for the potential benefit of lossless compression on those traces. Not surprisingly, we find that the information content is small in SNMP traces, higher in NetFLow traces, and extremely high in full packet level traces. More interestingly, we show that full packet header traces can be compressed in practice down to a minimum of $20 \%$ of their original size, and that the amount of compression is a function of the average flow size traversing that node: the larger the average flow size, the smaller the compression ratio.

Regarding traces collected at multiple monitoring points, we find that joint coding or compression of traces further reduces the marginally compressed traces at the individual monitors. Specifically, the joint compression ratio (or equivalently, the additional compression benefit brought by joint coding of traces) is low for SNMP or byte/packet count traces, higher for NetFlow or flowlevel traces, and higher still for packet-level traces. This means, for example, that joint coding is not really useful for SNMP data collected from different monitoring points and sent back to a NOC or central analysis station. Since SNMP data reporting takes little bandwidth anyway, it makes sense not to invest in sophisticated coding techniques in this case. However, NetFlow or packet header capture and reporting can require a significant amount of bandwidth and storage. Our results show that, in this case, joint coding techniques have the potential to significantly reduce those bandwidth and storage requirements.

Information-theoretic concepts and approaches have been used in the past to examine a wide variety of networking issues such as connectivity [18] and traffic matrix estimation [26], anomaly detection [16], and compact traffic descriptors $[8,24]$ for network dimensioning and QoS monitoring. However, to our knowledge, our attempt is the first to introduce a framework within which to address all the questions of interest here, namely trace coding, correlated and joint coding, and trace content at multiple time scales.

There has been work on trace compression, however it has been heuristic in nature. For example, work described in [14, 23] proposed heuristics based on storing and compressing packet headers collected at a single monitor along with timing information in the form of flow records. Trajectory sampling exhibits some elements in common with distributed compression of monitored data [7]. Also related is work in the area of in- verting sampled data, e.g., $[9,11]$. Indeed, sampling can be thought of as a form of lossy compression and these papers are concerned with decoding the resulting traces. There is also an extensive body of work produced within the sensor networking and distributed signal processing communities; see [4, 6] and their references for examples. However, much of it is in the context of abstract models of how data is produced, e.g., Gaussian random fields, and is not directly applicable in the domain of network monitoring.

The rest of the paper is structured in three parts. In Section 2 we present the various elements of our framework, including relevant concepts from information theory, our network flow-level model, and the network traces that will be used throughout the paper. In Section 3, we describe the first application of our framework, specifically we derive the information content of packet header traces collected at a single monitoring station and examine the benefits of trace compression in this case. In Section 4, we examine the more general problem of correlated, or joint, coding of trace captured at several monitoring stations. Section 5 then applies the model to determine the loss in information content when either flow-level (Net Flow) or byte count (SNMP) summary is applied to the full trace. Section 6 concludes the paper.

\section{Methodologies}

In this section we provide the foundation for the rest of the paper. We begin by reviewing several concepts needed from information theory (Section 2.1) that form the basis for exploring information content in network traces. This is followed with a description of the network flow model to which they will be applied (Section 2.2). The section ends with a review of a collection of network traces used to validate and parameterize the model (Section 2.3).

\subsection{Some concepts from Information The- ory}

We begin by introducing the concepts of entropy and entropy rate and their relation to data compression [5].

Definition 1 Shannon entropy. Let $X$ be a discrete random variable that takes values from $\chi$. Let $p(x)=$ $P(X=x), x \in \chi$. The entropy of $X$ is defined by

$$
H(X)=-\sum_{x \in \chi} p(x) \log _{2} p(x)
$$

Examples of $X$ in our context would be the flow size measured in packets, the flow identifier, and the packet/byte count in a one second interval. 
Now consider a stochastic process $X=\left\{X_{n}\right\}_{n=1}^{\infty}$ where $X_{n}$ is discrete valued.

Definition 2 Entropy Rate. The entropy rate of a discrete valued stochastic process $X$ is defined by

$$
H(X)=\lim _{n \rightarrow \infty} \frac{H\left(X_{1}, X_{2}, \ldots, X_{n}\right)}{n}
$$

when the limit exists.

The entropy rate represents the information rate conveyed by the stochastic process $X$. It provides an achievable lower bound on the number of bits per sample required for lossless compression of the process. With lossless compression, all of the information is completely restored when the file is uncompressed. In our context, an example might be the byte counts at a link over successive one second intervals.

Definition 3 Joint Entropy Rate. The joint entropy rate of a collection of many stochastic processes $\left\{X_{n}^{(i)}\right\}_{n=1}^{\infty}, i=1,2, \ldots, N$ is defined by

$$
\begin{aligned}
& H\left(X^{(1)}, X^{(2)}, \ldots, X^{(N)}\right) \\
= & \lim _{n \rightarrow \infty} \frac{H\left(\left(X_{1}^{(1)}, \ldots, X_{n}^{(1)}\right), \ldots,\left(X_{1}^{(N)}, \ldots, X_{n}^{(N)}\right)\right)}{n}
\end{aligned}
$$

when the limit exists.

The joint entropy rate represents the information rate conveyed by the joint stochastic process and is an achievable lower bound on the number of bits required per sample for the joint lossless compression of all the processes.

Let us place this in our context. Let $X_{i}$ be the header of the $i$-th packet and $M$ the size of the header. $\left\{X_{i}\right\}_{i=1}^{\infty}$ is a stochastic process representing packet headers. We are interested in quantifying the benefit gained from compressing a packet header trace gathered from one network monitor or traces collected at a set of network monitors.

Definition 4 Marginal Compression Ratio. Given stationary stochastic process $\left\{X_{i}\right\}_{i=1}^{\infty}$, the marginal compression ratio is defined as the ratio of the entropy rate and record size,

$$
\rho_{m}(X)=\frac{H(X)}{M}
$$

In the case of traces collected at multiple points within the network, we define:

Definition 5 Joint Compression Ratio. Given a collection of $N$ jointly stationary stochastic processes
$\left\{X_{i}^{(n)}\right\}_{i=1}^{\infty}, i=1,2, \ldots, N$, the joint compression ratio is defined as the ratio of the joint entropy rate and the sum of the entropy rates of the individual processes.

$\rho_{j}\left(X^{(1)}, X^{(2)}, \ldots, X^{(N)}\right)=\frac{H\left(X^{(1)}, X^{(2)}, \ldots, X^{(N)}\right)}{\sum_{i=1}^{N} H\left(X^{(i)}\right)}$

In the context of network trace compression, the joint compression ratio quantifies the potential benefits of jointly compressing the traces collected at several point in the network beyond simply compressing each trace independent of each other.

Although much of the time we will deal with discrete random variables, some quantities, such as interarrival times, are best approximated by continuous random variables. This necessitates the following definition.

Definition 6 Differential Entropy. Let $X$ be a continuous random variable with a density $f(X)$. The differential entropy of $X$ is defined by

$$
h(X)=-\int_{S} f(x) \log f(x) d x
$$

where $S$ is the support set of the random variable.

In reality, every variable is measured with finite resolution. With a resolution of $\epsilon=2^{-n}$, i.e., an $n$-bit quantization, a continuous random variable $X$ is represented by a discrete random variable $X^{\epsilon}$ and its entropy is approximately $h(X)+n$.

If $X$ follows an exponential distribution with rate $\lambda$, its differential entropy

$$
h(X)=-\int_{2}^{\infty} \lambda e^{-\lambda x} \log _{2}\left(\lambda e^{-\lambda x}\right) d x=\log _{2} \frac{e}{\lambda}
$$

With an n-bit quantization, the discrete entropy of $X$ is $H\left(X^{\epsilon}\right)=\log _{2} \frac{e}{\lambda}+n$. In the following, whenever there is no confusion, we use the notation $H(X)$ for a continuous random variable $X$ to represent its discrete entropy $H\left(X^{\epsilon}\right)$.

\subsection{Network Flow Model}

In this section we introduce a flow-based network model. We represent the network as a directed graph $G=$ $(V, E)$. Assume that flows arrive to the network according to a Poisson process with rate $\Lambda$ and $\Delta_{j}$ denotes the inter-arrival time between flow $j$ and $j-1$. Let $\Theta_{j} \in \mathcal{F}$ be the id of the $j$-th flow that arrives to the network. We assume that the route of a flow $f \in \mathcal{F}$ is fixed and classify flows into non-overlap flow classes, $\left\{\mathcal{F}_{i}, 1 \leq i \leq N\right\}$, such that all flows within a class share the same route in the monitored network. The route taken by flows in class $i$ is represented by an ordered set 


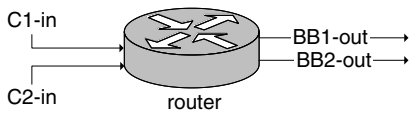

Figure 1: Experiment setup.

$R^{\langle i\rangle}=\left(v_{1}^{\langle i\rangle}, v_{2}^{\langle i\rangle}, \ldots, v_{\left.l^{\langle i\rangle}\right\rangle}^{\langle i\rangle}\right)$, where $v_{j}^{\langle i\rangle}$ is the $j$-th router traversed by a class $i$ flow and $l^{\langle i\rangle}$ is the path length. The flow arrival rate within class $i$ is $\Lambda^{\langle i\rangle}=\Lambda \times P\left(\Theta \in \mathcal{F}_{i}\right)$. When flow $j$ arrives, it generates $K_{j}$ packets. Packets within flow $j$ arrive according to some point process with inter-arrival times $\left\{\delta_{j, i}\right\}_{i=2}^{K_{j}}$, where $\delta_{j, i}$ is the inter-arrival time between the $i-1$ th and $i$ th packet of flow $j$. It is assumed that the first packet arrives at the same time as the flow. The behavior of packet arrivals in the network is described by the stochastic process $\left\{\left(\Delta_{j}, \Theta_{j}, K_{j},\left\{\delta_{j, i}\right\}_{i=2}^{K_{j}}\right)\right\}$.

In practice, network traces are collected at distributed network monitors. We are interested in how information is carried around by packets when they traverse distributed network monitors. As a starting point, we assume that there is no packet loss in the network and packets incur constant delay on each link: let $D_{(i, j), k}$ denote the delay that the $k$-th packet incurs while traversing the $k$-th link, $(i, j) \in E$, we assume that $D_{(i, j), k}=D_{(i, j)}$, $\forall k$. Delays are very small and losses non-existent in a well-provisioned network such as the Sprint network. Hence this is a reasonable assumption in many cases. In Section 4.3 we describe how these assumptions can be relaxed. For a node $v$ in the network, let $C^{(v)} \subseteq F$ denote the set of flows that pass through it. Since flows arrive to the network according to a Poisson process and the delay between any two nodes in the network is constant, flows arrive to node $v$ according to a Poisson process with rate $\Lambda^{(v)}=\Lambda \times P\left(\Theta \in C^{(v)}\right)$. The behavior of packet arrivals at node $v$ can be described by the stochastic process $\left\{\left(\Delta_{j}^{(v)}, \Theta_{j}^{(v)}, K_{j}^{(v)},\left\{\delta_{j, i}^{(v)}\right\}_{i=2}^{K_{j}^{(v)}}\right\}\right)$, where $\left\{\Delta_{j}^{(v)}\right\}$ is the sequence of inter-flow-arrival times at node $v$ that follows exponential distribution with rate $\Lambda^{(v)},\left\{\Theta_{j}^{(v)}\right\}$ is an i.i.d. sequence of flow ids seen by $v,\left\{K_{j}^{(v)}\right\}$ is an i.i.d, sequence of integer valued random variables that denote the number of packets in the $j$ th flow passing through $v$ and $\left\{\delta_{j, i}^{(v)}\right\}_{i=2}^{K_{j}^{(v)}}$ is the sequence of flow inter packet arrival times.

Figure 1 illustrates a simple scenario corresponding to a router with two incoming links and two outgoing links, each of which contains a monitor. There are four two hop paths and four flow classes traversing these paths. This is the setting within which the traces used for validation and parameterization of the model are collected. They are described in the next section.

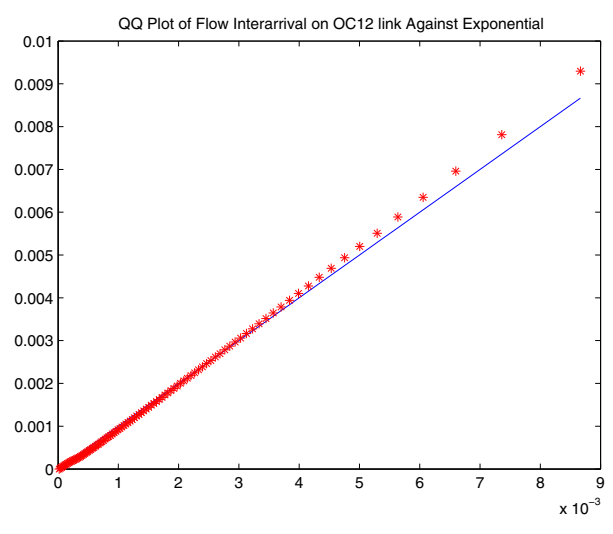

Figure 2: Flow arrival is Poisson

\subsection{Validation}

\subsubsection{Network Traces}

The data used here were collected as part of the full router experiment [13] on August 13, 2003. Packet traces were recorded at six interfaces, i.e. 12 links of one gateway router of the Sprint IP backbone network for 13 hours. We use mutually synchronized DAG cards [1] to record packet headers with GPS-precisioned 64bit timestamps from OC-3, OC-12 and OC-48 links [10]. The experiment setup is shown in Figure 1. In the remainder of this paper, we will focus on the traces collected over a one hour time period between 14:30 and 15:30 GMT on two incoming and two outgoing links. Table 1 summarizes the size of these traces and the link utilizations.

We choose this set of traces because it captures a mix of customer to gateway and gateway to backbone traffic. Customer traffic largely goes to one of the two backbone links. It is an ideal first step to analyze information redundancy without being overwhelmed by complicated routing. Furthermore, it is a representative set of traffic data, because there are over 500 similarly configured gateway routers in Sprint's global network. The shortcoming is that we do not have any backbone to backbone traffic recorded at the same time. This will be considered in the future work.

We further select the three most highly utilized links from the set to use in the remainder of the paper. BB1out, BB2-out are from two OC-48 linecards connecting to two backbone routers (BB1 and BB2). $\mathrm{C} 1$-in and $\mathrm{C} 2-$ in are from two OC-3 linecards connecting to transpacific customers. We use the full packet trace to deduce an SNMP-like utilization trace (refered to as utilization trace from now on) and an unsampled raw Netflow trace. This is done so that different network measurement techniques can be compared for the same time duration without any possible measurement errors con- 


\begin{tabular}{|r|r|r|r|}
\hline Data Set & duration & \#packets & Average rate \\
\hline C1-in & 1 hour & $44,598,254$ & 53Mbps \\
C2-in & 1 hour & $123,542,790$ & $73 \mathrm{Mbps}$ \\
BB1-out & 1 hour & $71,115,632$ & 69Mbps \\
BB2-out & 1 hour & $105,868,070$ & $82 \mathrm{Mbps}$ \\
\hline
\end{tabular}

Table 1: Trace description and stats.

tributed by SNMP or Netflow. The utilization trace is computed as byte-count per second rather than per $5 \mathrm{~min}$ utes as a normal SNMP trace. This is done to increase the number of data points and minimize estimation error in the calculation. We simulate Netflow by creating an unsampled Netflow trace from the packet trace, because we prefer to not take into account packet sampling's effect on measurement at this stage.

The full packet traces described above are used to validate the Poisson assumptions made in the flow model. Figure 2 depicts a QQ plot of the empirical inter-flow arrival time distribution with respect to an exponential distribution with the same average. We observe visually a good match for the flow inter-arrival times associated with link C1-in. This is also the case with the other flow arrival traces and is consistent with observations made elsewhere [12]. We compute empirical entropy on the traces described above using empirical marginal probability distributions.

\section{Application: Single Point Trace Com- pression}

All packet monitoring poses tremendous challenges to the storage subsystems due to the high volume of current network links. We use the information theoretic approach to identify and quantify the potential benefit of network trace compression based on the network flow model. In this section, we focus on full trace collection at a single monitor such as an IPMON system [10]. We start with calculating the information content in traces made of packet headers. It will become clear that the temporal correlation resulted from the flow structure leads to considerable marginal compression gain. We then show by empirical study that other fields in IP header will contain no/very small amount of information. We conclude this section with guidelines for the development of practical network trace compression algorithms. The next section concerns the simultaneous collection of traces at multiple monitors distributed throughout a network.

\subsection{Entropy of Flow-based Trace}

In the IPMON style full packet header trace capture system, The IP header and additional TCP/UDP header of each packet is stored in the trace. Here we only concern with the information content in the IP header, and leave TCP/UDP header for future work (except the port fields). We start with calculating the information content in packet time-stamp and 5-tuple flow ID.

The total length of an uncompressed timestamp is 64. The behavior of packet arrivals in the network is described by the stochastic process $\left\{\left(\Delta_{j}, \Theta_{j}, K_{j},\left\{\delta_{j, i}\right\}_{i=2}^{K_{j}}\right)\right\}$ (to avoid triviality, we assume $\left.P\left(K_{j}>1\right)=1\right)$. We are interested in determining the minimum number of bits required to represent each flow. If we assume flow inter-arrival $\left\{\Delta_{j}\right\}$, flow Id $\left\{\Theta_{j}\right\}$ and packet inter-arrivals within a flow $\left\{\delta_{j, i}\right\}_{i=2}^{K_{j}}$ are pairwise independent, on average we need a number of bits per flow equal to $H(\Phi)$ where

$$
H(\Phi)=H(\Theta)+H(\Delta)+H\left(\left\{\delta_{i}\right\}_{i=2}^{K}\right),
$$

where $H\left(\left\{\delta_{i}\right\}_{i=2}^{K}\right)$ denotes the information content in packet inter-arrivals within a flow. It can be shown that

$$
\begin{aligned}
H\left(\left\{\delta_{i}\right\}_{i=2}^{K}\right) & =H(K)+H\left(\left\{\delta_{i}\right\}_{i=2}^{K} \mid K\right) \\
& =H(K)+\sum_{k=2}^{\infty} P(K=k) H\left(\left\{\delta_{i}\right\}_{i=2}^{k} \mid K=k\right) \\
& \leq H(K)+\sum_{k=2}^{\infty} P(K=k)(k-1) H\left(\delta_{i} \mid K=k\right) \\
& \leq H(K)+(E[K]-1) H(\delta),
\end{aligned}
$$

where $\delta$ represents the inter-arrival time between randomly picked adjacent packet pairs from all flows. Inequality (5) is an equality if packet inter-arrival times within a flow, $\left\{\delta_{i}\right\}$, are i.i.d. sequence. Inequality (6) is an equality if packet inter-arrival times are independent of flow size $K$.

Fig. 3 shows there is in fact a strong correlation between flow size and inter-packet arrivals time within a flow. Large flows with many packets tend to have smaller inter-packet arrival times. This suggests there is opportunity in further compressing the inter-packet arrival time within a flow. However the Inequality (6) provides us with an upper bound in compression ratio.

The per-flow information consists of two parts: one part is timing information about the flow arrival and flow ID, which is shared by all packets in the flow; the other part consists of all the packet inter-arrival information, which grows sub-linearly with the number of packets within the flow if we assume packet inter-arrivals are dependent. (Note: If packet inter-arrival times are independent it grows linearly.) The information rate per unit time is then $\Lambda H(\Phi)$. 


\begin{tabular}{|r|r|r|r|r|r|r|r|r|}
\hline Trace & $\begin{array}{r}H(\Delta) \\
\text { bin }=8 \mu \mathrm{s}\end{array}$ & $\begin{array}{r}H(K) \\
\text { bin=2pkts }\end{array}$ & $\begin{array}{r}H(\delta) \\
\text { bin=128 } \mu \mathrm{s}\end{array}$ & $H(\Theta)$ & $E(K)$ & $H($ total $)$ & $\rho(\Phi)$ & $\begin{array}{r}\text { Compression } \\
\text { Algorithm }\end{array}$ \\
\hline C1-in & 8.8071 & 3.2168 & 9.7121 & 104 & 21.0039 & 706.3772 & $\mathbf{0 . 2 0 0 2}$ & $\mathbf{0 . 6 4 2 5}$ \\
\hline BB1-out & 7.6124 & 2.4688 & 12.1095 & 104 & 20.4825 & 736.1722 & $\mathbf{0 . 2 1 3 9}$ & $\mathbf{0 . 6 5 7 4}$ \\
\hline BB2-out & 7.1594 & 2.7064 & 12.4824 & 104 & 18.7890 & 689.9066 & $\mathbf{0 . 2 1 8 6}$ & $\mathbf{0 . 6 6 5 7}$ \\
\hline
\end{tabular}

Table 2: Comparision of entropy calculations and real compression algorithm gain

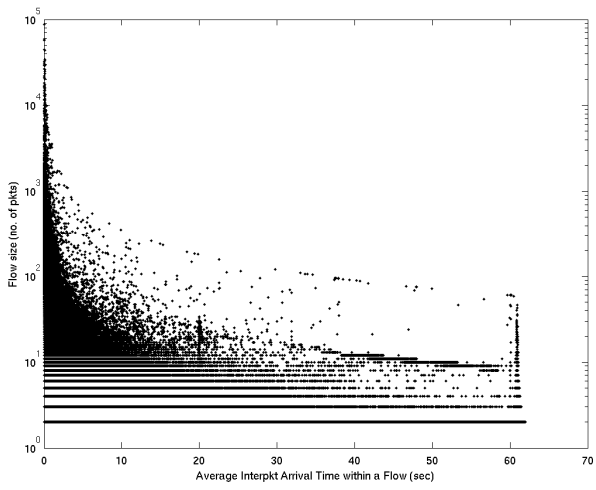

Figure 3: Flow size correlation with inter-packet arrival time within a flow. Larger flow size correspond to shorter inter-packet arrival times.

There exists other fields in the IP header as well, such as TOS, datagram size, etc. A detailed study in [19] shows that they carry little information content in the framework of the flow model, and can be modeled similarly to the packet inter-arrival time within a flow. We therefore take the simplified approach and only consider the timestamp field and the flow ID field in this paper.

\subsection{Marginal Compression Ratio}

In practice, traces are collected at individual nodes. For a node $v$ in the network, we need a number of bits per flow equal to $H\left(\Phi^{(v)}\right)$ where

$$
\begin{aligned}
H\left(\Phi^{(v)}\right) & \leq H\left(\Theta^{(v)}\right)+H\left(\Delta^{(v)}\right)+H\left(K^{(v)}\right) \\
& +\left(E\left[K^{(v)}\right]-1\right) H\left(\delta^{(v)}\right)
\end{aligned}
$$

As before the inequality becomes equality when $\left\{K^{(v)}\right\}$ is independent of $\left\{\delta_{f, i}^{(v)}\right\}$ and the latter sequence is iid.

The information rate per unit time is then $\Lambda^{(v)} H\left(\Phi^{(v)}\right)$ at node $v$. In the absence of compression, each flow requires on average $(104+64) E\left[K^{(v)}\right]$ bits with 104 bits to encode the 5 -tuple flow identifier and 64 bits for timestamps of packet arrivals.

Now we can answer the question: what is the maximum benefit that can be achieved through compression?
From $\Phi^{(v)}$, we have a marginal compression ratio

$$
\rho\left(\Phi^{(v)}\right)=\frac{H\left(\Phi^{(v)}\right)}{168 * E\left[K^{(v)}\right]}
$$

The compression ratio $\rho\left(\Phi^{(v)}\right)$ provides a lower bound on what can be achieved through lossless compression of the original network trace. From (7) and (8), the marginal compression ratio at a node is a decreasing function of $E\left[K^{(v)}\right]$, the average size of flows traversing that node. Since the information in flow ID $\Theta^{(v)}$ and flow arrival $\Delta^{(v)}$ is shared by all packets in the flow, the larger the average flow size, the smaller the per-packet share, therefore the smaller the compression ratio. When $E\left[K^{(v)}\right]$ is large, the compression ratio is bounded from below by $\left(E\left[H\left(\delta^{(v)}\right)\right]\right) / 168$, which is an indication of how compressible the packet inter-arrival time is in average. (Note: this bound results from the assumption that packet inter-arrival times within a flow are independent. If there is correlation between packet inter-arrival times, a tighter bound can be derived to explore the correlation.) Therefore, the marginal compression ratio for long flows is determined by the compression ratio of packet interarrival times.

\subsection{Results}

We summarized the marginal compression ratio for a single trace in Table 2. We compare the compression upperbound from the flow model entropy calculation and a practical trace compression algorithm. The trace compression software is an implementation of the schemes introduced in [14]. The practical algorithm uses a flowbased compressed scheme very similar to the flow model in section 2.2. It does, however, record additional IPID, datagram size and TCP/UDP fields in each packet of a flow.

For the three traces we considered, the entropy calculation suggests a bound of compression ratio $20.02 \%$ to $21.86 \%$ of the original trace. However the practical scheme only compresses $64.25 \%$ to $66.57 \%$ of the original trace. The most significant algorithm difference is that this scheme keeps a fixed packet buffer for each flow, therefore records very long flows as many smaller flows. This is done for the ease of decompression and quickly 
count for very long flows, avoiding only outputting long flows after they end. This could result in long flows being broken down to many small flows and duplicate the flow record many times, therefore reducing the compression benefits.

\section{Application: Joint Trace Coding}

The fact that one flow traverses multiple monitors introduces spatial correlation among traces collected by distributed monitors. We calculate the joint entropy of network traces which serves as the lower bound for distributed traces compression. To account for network uncertainties, such as packet loss and random delay, we incorporate additional terms to characterize network impairment.

\subsection{Joint Compression Ratio}

We are interested in quantifying how well marginal compression comes to achieving the entropy rate of the network. We have

$$
\rho_{j}(\Phi)=\frac{\Lambda H(\Phi)}{\sum_{v \in V} \Lambda^{(v)} H\left(\Phi^{(v)}\right)}
$$

where the numerator is the lower bound on joint compression and the denominator is the lower bound of marginal compression of each trace separately. The joint compression ratio $\rho_{j}$ shows the benefit of joint compression.

The entropy of a flow record of class $i$ can be calculated as $H\left(\Phi^{\langle i\rangle}\right)$. The information rate generated by flow class $i$ is $\Lambda^{\langle i\rangle} H\left(\Phi^{\langle i\rangle}\right)$. Therefore, the network information rate is:

$$
\Lambda H(\Phi)=\sum_{i=1}^{N} \Lambda^{\langle i\rangle} H\left(\Phi^{\langle i\rangle}\right)
$$

At node $v$, denote by $D^{\langle v\rangle}$ the set of flow classes traversing it. The total information rate at $v$ is

$$
\Lambda^{(v)} H\left(\Phi^{(v)}\right)=\sum_{i \in D^{\langle v\rangle}} \Lambda^{\langle i\rangle} H\left(\Phi^{\langle i\rangle}\right)
$$

Plugging (10) and (11) into (9), we have

$$
\begin{aligned}
\rho_{j} & =\frac{\sum_{i=1}^{N} \Lambda^{\langle i\rangle} H\left(\Phi^{\langle i\rangle}\right)}{\sum_{v \in V} \sum_{i \in D^{\langle v\rangle}} \Lambda^{\langle i\rangle} H\left(\Phi^{\langle i\rangle}\right)} \\
& =\frac{\sum_{i=1}^{N} \Lambda^{\langle i\rangle} H\left(\Phi^{\langle i\rangle}\right)}{\sum_{i=1}^{N} l^{\langle i\rangle} \Lambda^{\langle i\rangle} H\left(\Phi^{\langle i\rangle}\right)}
\end{aligned}
$$

It suggests that the joint compression ratio is inversely proportional to a weighted average of the number of

\begin{tabular}{|c|c|}
\hline & Joint Compression Ratio \\
\hline$\rho(\mathrm{C} 1$-in, BB1-out, $\mathrm{C} 2$-in, BB2-out $)$ & $\mathbf{0 . 5}$ \\
\hline$\rho(\mathrm{C} 1$-in to BB1-out $)$ & $\mathbf{0 . 8 6 4 9}$ \\
\hline$\rho(\mathrm{C} 1$-in to BB2-out $)$ & $\mathbf{0 . 8 7 0 2}$ \\
\hline$\rho(\mathrm{C} 2$-in to BB1-out $)$ & $\mathbf{0 . 7 1 2 5}$ \\
\hline$\rho(\mathrm{C} 2$-in to BB2-out $)$ & $\mathbf{0 . 6 6 7 9}$ \\
\hline
\end{tabular}

Table 3: Joint trace compression ratio

monitors traversed by flows. Intuitively, all monitors traversed by a flow collect redundant information for that flow. The longer the flows paths, the higher the spatial correlation in distributed packet header traces, the bigger the potential gain of distributed trace compression.

\subsection{Entropy Results}

According to (9), the joint compression ratio for all the links traversing through our router is simply 0.5 , since the path length $(l)$ is 2 for each flow class. We're also interested in knowing the joint compression ratio of a path connecting any two links. For example, let us denote the flow classes $\mathrm{C} 1$-in to BB1-out as A, C1-in to BB2-out as $\mathrm{B}$, and $\mathrm{C} 2$-in to $\mathrm{BB} 1$-out as $\mathrm{C}$, the compression ratio for the path through links $\mathrm{C} 1$-in and $\mathrm{BB} 1-$ out is:

$$
\begin{aligned}
\rho & =\frac{H(B B 1-\text { out }, C 1-\text { in })}{H(B B 1-\text { out })+H(C 1-\text { in })} \\
& =\frac{\Lambda^{A} H(A)+\Lambda^{B} H(B)+\Lambda^{C} H(C)}{2 \Lambda^{A} H(A)+\Lambda^{B} H(B)+\Lambda^{C} H(C)}
\end{aligned}
$$

The results in Table 3 show a substantial $50 \%$ to $87 \%$ joint compression ratio over multiple monitoring points. The ratio is lower (namely 0.5 ) when computed across all monitors from the same router, and higher (namely 0.6679 to 0.8702 ) when computed over only two links. The savings come from a significant amount of shared flows in these monitoring points. This suggests that if we can successfully share information at the correlated monitoring points and perform joint coding, the potential savings will be very significant. In practice, it could be difficult to devise a perfect coding scheme for the entire network. We leave this for a future direction.

\subsection{Network Impairments}

Till now we have assumed that link delays are constant and that there are no packet losses. These can be accounted for by augmenting the expression for the flow entropy to account for these. Take the case where link delays are not constant as an example. Due to variable link delays, the inter-arrival time of two adjacant packets 
within a flow varies when they traverse the network. Link delays may also induce packet reordering. To fully capture the timing information of all packets in the network, one will have to record not only the packet inter-arrival time within a flow at their network entry point, but also the packet delays on all links. Suppose for now that the delay sequences $\left\{D_{(i, j), k}\right\}_{k=1}^{\infty},(i, j) \in E$ are mutually independent sequences of iid rvs. Then $H(\Phi)$ becomes

$$
H(\Phi)=H_{w}(\Phi)+H_{n}(\Phi)
$$

where $H_{w}(\Phi)$ is given by (2) and $H_{n}(\Phi)$ is

$$
H_{n}(\Phi)=E[K]\left(H\left(D_{(i, j)}\right)\right)
$$

Note that in the case that link delays are correlated, this provides an upper bound. Figure 4 plots the distribution of the single hop packet delay on an operational router in Sprint Network. The delay is quantized with resolution of one micro-second, which corresponds to the accuracy of clock synchronization between two link monitors. From the figure, although the delay is not constant, it has skewed distribution. The empirical entropy is 4.2615 , which means, in average, we need no more than 5 bits per packet to represent the variable packet delays across this router. Furthermore, we expect that packet delays are temporally correlated. By exploiting the temporal correlation, one can using even smaller number of bits to represent packet delays.

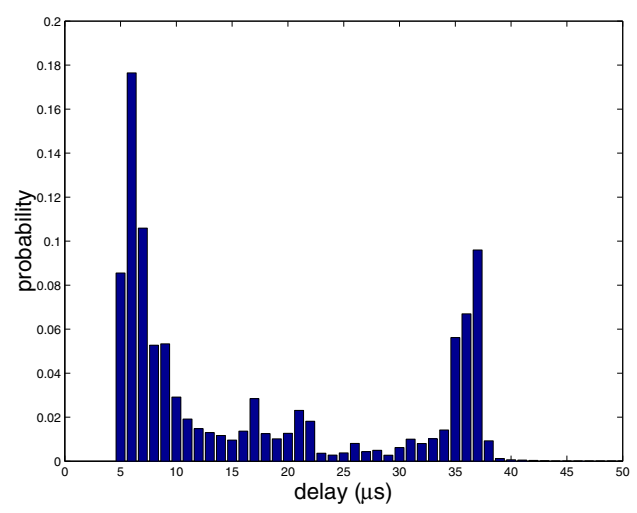

Figure 4: Single Hop Delay on Operational Router

The common belief is there is very little loss in large operational backbone networks, since they are usually over-provisioned. However, we expect that losses will introduce an additional term in the expression for $H_{n}()$. In particular, when losses are rare, they are likely to show up in the form of the entropy of a Bernoulli process, one for each link in the network. This will be the subject of future investigation.

Another impairment occurs when the routes change within the network. Fortunately, route changes are infrequent [25]. When they do occur, the changes must be recorded in the traces. However, they increase the information rate per unit time by an insignificant amount.

\subsection{Development of Joint Compression Al- gorithms}

Our models predict there is considerable gain to conduct joint packet header trace compression. It opens up the question of how one can develop algorithms to achieve the predicted joint compression ratio.

One direction is to develop a centralized algorithm to compress traces collected on distributed monitors. This requires all monitors to send their traces to a centralized server, which will generate a compressed aggregate trace. This approach will incur communication overhead in transmitting all those traces to the server. To reduce the communication overhead, traces can be aggregated on their ways to the server. Our spatial correlation model can be used to optimally organize the transmission and compression of traces. This is similar to the problem of joint routing and compression in sensor networks [22].

Alternatively, one can develop a distributed trace compression algorithm. Distributed data compression [5] aims at compressing correlated sources in a distributed way and achieving the gain of joint compression. How monitors compress packet traces without exchanging packet headers remains to be a challenging problem. It may also be possible to borrow ideas from trajectory sampling [7] to design joint compression algorithms.

\section{Application: Information Content of Dif- ferent Measurement Methods}

In this section we apply information theory to evaluate the information gained from monitoring a network at different granularities. In addition to the packet header monitoring paradigm as exemplified by IPMON [14], we examine two other typical monitoring options. The most widely used monitoring method is SNMP utilization data collection [15], which is provided by all routers by default and has very low storage requirement. A second option is Cisco NetFlow [2] and its equivalent, which requires better router support and more demanding storages and analysis support. Clearly packet header monitoring is the most demanding and requires the greatest amount of resources, $\mathrm{v}$ however it provides the most comprehensive amount of information about the network. We first adopt the flow modelling of the network from section 2.2 to study both NetFlow and SNMP monitoring options. We then explore the quantitative difference among these methods using entropy calculation applied to the models. 


\subsection{A Model for NetFlow}

Recording complete packet header traces is expensive and, thus, not commonly done. Cisco NetFlow is provided by some Cisco linecards to summarize flows without capturing every packet header. A raw NetFlow trace consists of a set of flow records. Each flow record includes the start time and duration of the flow, and the size of the flow (number of packets, bytes). In this section we explore the potential benefits of compression, both at a single monitor and across multiple monitors for this type of trace. In practice, NetFlow can sample packets on high volume links. For this paper, we consider the case where all packets are observed to derive flow records, hence avoiding inaccurate representation of flows from packet sampling described in [11] and [3].

A NetFlow record consists of an arrival time (flow inter-arrival time), flow ID, flow size, and the flow duration. Very similar to the flow model in Section 3.1, the average number of bit per flow record is

$$
H(\Psi)=H(\Delta)+H(\Theta)+H(K)
$$

We assume the five tuple flow ID is not compressed since it does not repeat in the capturing window.

In practice, each node individually turns on NetFlow and captures information for flows traversing it. The entropy of a NetFlow record at node $v$ can be calculated as

$$
H\left(\Psi^{(v)}\right)=H\left(\Delta^{(v)}\right)+H\left(\Theta^{(v)}\right)+H\left(K^{(v)}\right)
$$

Similar to Section 3.1, we can calculate the entropy of a NetFlow record of flow class $i$ as $H\left(\Psi^{\langle i\rangle}\right)$. The joint compression ratio of NetFlow traces can be calculated as

$\rho_{j}(\Psi)=\frac{\Lambda H(\Psi)}{\sum_{v \in V} \Lambda^{(v)} H\left(\Psi^{(v)}\right)}=\frac{\sum_{i=1}^{N} \Lambda^{\langle i\rangle} H\left(\Psi^{\langle i\rangle}\right)}{\sum_{i=1}^{N} l^{\langle i\rangle} \Lambda^{\langle i\rangle} H\left(\Psi^{\langle i\rangle}\right)}$

The joint compression ratio is again inversely proportional to a weighted average of the number of monitors traversed by a flow. This suggests that NetFlow traces without sampling preserve the spatial correlation contained in full flow level packet trace. We show the joint compression ratio results based on our trace in Table 5 .

So far, we assume NetFlow processes all the packets in all the flows. In a real network environment, both the number of flows and the number of packets generated by those flow are large. NetFlow can employ flow sampling, where only a fraction of flows are monitored, and packet sampling, where only a fraction of packets are counted, to reduce its cost in computation and memory, etc. Flow sampling will proportionally reduce the amount of flow information one obtained from the network. If two monitors sample flows independently with probability $p$, the chance that one flow gets sampled at both monitors is $p^{2}$, which leads to a reduced spatial correlation in NetFlow traces collected by these two monitors. Packet sampling will introduce errors in flow size and flow duration estimation. Furthermore, NetFlow records collected by two monitors for the same flow will be different. By aggregating distributed NetFlow traces, one can obtain more accurate flow information. We will extend our NetFlow model to account for flow sampling and packet sampling in future work.

\subsection{A Model for SNMP}

In this section we study the information content of SNMP measurements. By SNMP measurements, we mean packet/byte counts over fixed intervals of time. We begin with the flow model from Section 2.2. Let $\left\{\left(R_{1}(t), \ldots, R_{|E|}\right): t \geq 0\right\}$, be the rate process associated with the network. In other words, $R_{i}(t)$ is the packet rate on link $i \in E$ at time $t \geq 0$. We will argue that it can be modeled as a multivariate Gaussian process and will calculate its associated parameters.

Let $\left\{R^{j}(t): t \geq 0\right\}$ be the rate process associated with flow class $j \in[1, N]$, in other words, $R^{j}(t)$ is the rate at which flows within class $j \in[0, N]$ generate packets at time $t \geq 0$. Note that the processes associated with the different flow classes are independent. We have

$$
R_{i}(t)=\sum_{j \in \mathcal{S}(i)} R^{j}(t), \quad i \in E
$$

Here $\mathcal{S}(i) \subseteq[1, N]$ is the set of flow classes, whose flows pass through link $i \in E$. If we define the routing matrix $\left\{A_{i j}, 1 \leq i \leq|E|, 1 \leq j \leq M\right\}$ such that $A_{i j}=1$ if link $i$ is on the path of flow class $j$ and $A_{i j}=0$ otherwise, we have

$$
R_{I}=A R^{J}
$$

where $R_{I}=\left\{R_{i}, 1 \leq i \leq|E|\right\}$ is the rate vector on all network links and $R^{\bar{J}}=\left\{R^{j}, 1 \leq j \leq N\right\}$ is the rate vector of all flow classes.

It has been observed that the traffic rate on high speed link tends to be Gaussian [17]. We further assume that the rate processes associated with the flow classes are independent Gaussian processes. Figure 5 plots the distribution of the traffic rate of one flow class in the trace under study. The Q-Q plot against Gaussian distribution shows a good match. The rate vector of all flow classes follows a multi-variate Gaussian distribution with mean $\mu^{J}=E\left[R^{J}\right]=\left\{\mu^{j}\right\}$ and covariance $K^{J}=\operatorname{cov}\left(R^{J}\right)=$ $\left\{K_{m n}^{J}\right\}$, where $K_{m n}^{J}=\sigma_{j}^{2}$ if $m=n=j$ and 0 otherwise. Consequently, the link rate vector is also a multivariate Gaussian process with

$$
\begin{aligned}
\mu_{I} & =E\left[R_{I}\right]=A \mu^{J} \\
K_{I} & =\operatorname{cov}\left(R_{I}\right)=A K^{J} A^{T}
\end{aligned}
$$




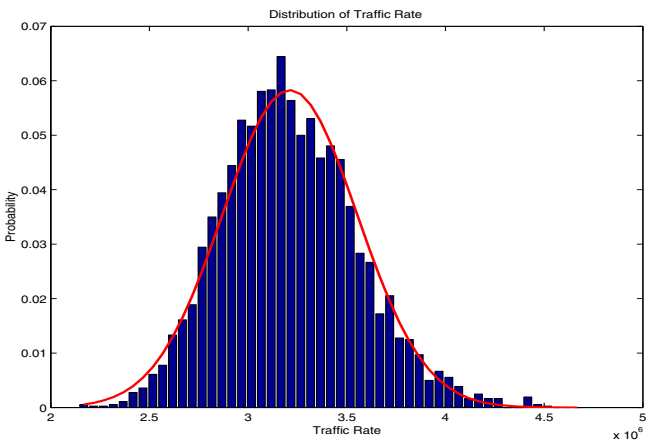

(a) Empirical Distribution

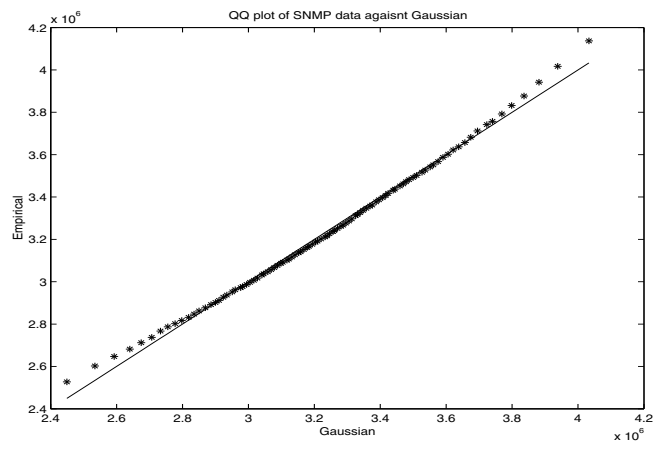

(b) QQ Plot Against Gaussian Distribution

Figure 5: Marginal Distribution of SNMP Data

\subsubsection{Entropy in SNMP Data}

According to [5], the marginal entropy of SNMP data collected on link $i$ can be calculated as:

$$
h\left(R_{i}\right)=\frac{\log 2 \pi e \sum_{j=1}^{N} A_{i j} \sigma_{j}^{2}}{2}=\frac{\log 2 \pi e \sum_{j \in \mathcal{S}(i)} \sigma_{j}^{2}}{2}
$$

The joint entropy of SNMP data collected on link $i$ and $j$ can be calculated as:

$$
\begin{aligned}
h\left(R_{i}, R_{l}\right) & =\frac{\log \left\{(2 \pi e)^{2}\left|\left[\begin{array}{l}
A_{i \cdot} \\
A_{l \cdot}
\end{array}\right] K^{J}\left[A_{i \cdot}^{T} \quad A_{l \cdot}^{T}\right]\right|\right\}}{2} \\
& =\frac{\log \left\{(2 \pi e)^{2}\left(K_{11} K_{22}-K_{12}^{2}\right)\right\}}{2},
\end{aligned}
$$

where $A_{i}$. is the $i$-th row vector in routing matrix $A$ and $K_{11}=\sum_{j \in \mathcal{S}(i)} \sigma_{j}^{2}, K_{22}=\sum_{j \in \mathcal{S}(j)} \sigma_{j}^{2}$ and $K_{21}=$ $\sum_{j \in \mathcal{S}(i) \cap \mathcal{S}(j)} \sigma_{j}^{2}$. Therefore

$$
\begin{aligned}
h\left(R_{i}, R_{l}\right) & =\frac{\log \left\{(2 \pi e)^{2} K_{11} K_{22}\right\}}{2}+\frac{\log \left\{1-\frac{K_{12}^{2}}{K_{11} K_{22}}\right\}}{2} \\
& =h\left(R_{i}\right)+h\left(R_{l}\right)+\frac{\log \left(1-\rho_{i l}^{2}\right)}{2},
\end{aligned}
$$

where $\rho_{i l}=\frac{K_{12}}{\sqrt{K_{11} K_{22}}}$ is the covariance coefficient between $R_{i}$ and $R_{l}$. The mutual information between SNMP data on link $i$ and link $l$ is

$$
\begin{aligned}
I\left(R_{i}, R_{l}\right) & =h\left(R_{i}\right)+h\left(R_{l}\right)-h\left(R_{i}, R_{l}\right) \\
& =-\frac{\log \left(1-\rho_{i l}^{2}\right)}{2},
\end{aligned}
$$

which could be small even if $\rho_{i l}$ is close to one. For example, suppose there are 900 flows traversing both link $i$ and link $l$. In addition to those common flows, links $i$ and $l$ each have their own 100 flows. If we assume all flows are statistically homogeneous, then we will have $\rho_{i l}=0.9$, which indicates $R_{i}$ and $R_{l}$ are highly correlated. However, according to (22), the mutual information between $R_{i}$ and $R_{l}$ is only around 1.2 bits. This suggests there is not much gain in doing joint compression of SNMP data.

\subsubsection{How much information does SNMP contain about traffic matrices?}

For the purpose of traffic engineering, it is important to characterize the traffic demand between all pairs of network ingress and egress points, or the Traffic Matrix (TM). Each element in the TM corresponds to the traffic rate of the flow class which goes from the ingress point to the egress point. Such information is normally not easy to access. Network operators can instrument each router to record SNMP data on each link, which is the sum of traffic rates of all flow classes traversing that link. It is challenging to infer the TM, equivalently the flow rate vector, based on the SNMP rate vector [26]. Our framework provides a way to quantify the amount of information about a TM that one can obtain from SNMP data.

Essentially, the information content in the flow rate vector (or TM) is

$$
\begin{aligned}
h\left(R^{J}\right) & =\sum_{j \in[0, N]} h\left(R^{j}\right) \\
& =\sum_{j \in[0, N]} \frac{1}{2} \log 2 \pi e \sigma_{j}^{2} .
\end{aligned}
$$

Since the link rate vector is a linear combination of the flow rate vector, we will always have

$$
h\left(R_{I}\right) \leq h\left(R^{J}\right) .
$$

Furthermore, under the Gaussian assumption, the information content in the link rate vector can be calculated 
as

$$
\begin{aligned}
h\left(R_{I}\right) & =\frac{1}{2} \log \left\{(2 \pi e)^{|E|}\left|K_{I}\right|\right\} \\
& =\frac{1}{2} \log \left\{(2 \pi e)^{|E|}\left|A K^{J} A^{T}\right|\right\} \\
& \leq h\left(R^{J}\right)=\frac{1}{2} \log \left\{(2 \pi e)^{N}\left|K^{J}\right|\right\}
\end{aligned}
$$

The gap in (29) is determined by both the routing matrix $A$ and the variance in the traffic rates of flow classes $\left\{\sigma_{j}^{2}\right\}$.

Note: For some routing matrices, the rows of $A$ are $d e$ pendent, i.e., some link rate $R_{l}$ is a linear combination of some other link rates. In this case, $R_{l}$ contributes no new information to the link rate vector; consequently, we should only include all independent row vectors of $A$ in (28) to calculate the entropy of the whole link rate vector. We will illustrate this through an example in Section5.3.

\subsubsection{Entropy Rate in SNMP}

In this section, we study the entropy rate in SNMP data by taking into account the temporal correlation in traffic rate processes. We have observed that the marginal link rate vector is well characterized as a multivariate Gaussian random variable. Consequently the link rate vector process over time is a stationary multivariate Gaussian process with mean $\mu_{I}$ given by (19) and covariance matrix at time lag $h, \Gamma_{I}(h)$, to be determined next. In order to simplify the discussion, we ignore link delays. These can be accounted for, but at the cost of some obfuscation. We have

$$
\Gamma_{I}(h)=A \times \Gamma^{J}(h) \times A^{T},
$$

where $\Gamma_{m n}^{J}(h)=G^{j}(h)$ if $m=n=j$ and 0 otherwise, and $G^{j}(h)$ is the covariance associated with the class $j$ rate process, $j \in[1, N]$. To obtain $\Gamma^{J}(h)$, we return to the original flow model and recognize that each flow class can be modeled as an $\mathrm{M} / \mathrm{G} / \infty$ queue and that we can apply known results regarding the autocorrelation function of its buffer occupancy process, [21]. If $T^{j}$ denotes the duration of a class $f$ flow and $\hat{T}^{j}$ denotes the forward recurrence time associated with $T^{j}, j \in[1, N]$, then

$$
G^{j}(h)=\Lambda^{j} E\left[T^{j}\right] \operatorname{Pr}\left[\hat{T}^{j}>|h|\right], \quad h \geq 0
$$

The distribution of $\hat{T}^{j}$ is

$$
\operatorname{Pr}\left[\hat{T}^{j}>h\right]=\frac{1}{E\left[T^{j}\right]} \int_{h}^{\infty} \operatorname{Pr}\left[T^{j}>x\right] d x, \quad h \geq 0
$$

Suppose that we sample over intervals of length $\tau$. Then we can use a discrete time version of the model where

$$
R_{k}^{j}=\int_{(k-1) \tau}^{k \tau} R^{j}(t) d t, \quad 1 \leq j \leq N
$$

Note that $\left\{R_{k}^{j}: k=1, \ldots\right\}$ is a discrete time Gaussian process with mean $\mu^{j} \tau$ and covariance approximately equal to $\Gamma^{\tau}(h)=\Lambda^{j} E\left[T^{j}\right] \operatorname{Pr}\left[\hat{T}^{j}>|h \tau|\right]$, $h=0,1,2, \ldots$. The discrete time counterparts for the rate process at the routers can be defined in a similar manner. The entropy rate of $\left\{R_{k}^{j}\right\}$ is given as, [5, Theorem 9.4.1]

$$
\begin{aligned}
h\left(\left\{R_{k}^{j}\right\}\right) & =\lim _{n \rightarrow \infty} \frac{1}{n} \frac{1}{2} \log (2 \pi e)^{n}\left|K_{n}^{j}\right|, \\
& =\frac{1}{2} \log (2 \pi e)+\lim _{n \rightarrow \infty} \frac{1}{2 n} \log \left|K_{n}^{j}\right|
\end{aligned}
$$

where $K_{n}^{j}$ is the $n \times n$ covariance matrix with elements $\Gamma^{\tau}(h), h=0,1, \ldots, n-1$. Finally, the entropy rate of the system is $\sum_{j=1}^{N} h\left(\left\{R_{k}^{j}\right\}\right)$.

\subsubsection{SNMP Evaluation}

The SNMP model is derived from the flow model and represents a summary of flow information. We evaluate the SNMP model by comparing the model derived entropy with an empirical entropy estimation.

The SNMP data is a per second utilization summarized from our trace data, instead of real per five minute data collected from the field. This is done to provide compatibility with the other monitoring methods. The empirical entropy estimation for each individual link is based on calculating empirical probability distribution function from the SNMP data, with a bin size of 50,000 bytes/sec. We use the BUB entropy estimator [20] with the PDF to derive the entropy. The BUB estimator does well when the number of available samples is small compared to the number of bins, as is the case here. It also provides an error bound for the estimation. For joint entropy such as $\mathrm{H}$ (BB1-out, C1-in), we compute the joint probability distribution of each SNMP data pair at time $t$ : (BB1-out(t), C1-in(t)), then compute the entropy using the BUB function. In Table 4 we verify that the entropy of the SNMP data derived from the Gaussian model indeed matches well with the empirical calculations.

\subsection{Results}

We present results on the comparison of the three monitoring options in terms of quantitative storage difference and distributed compression savings. The joint compression ratio indicates whether two links share a strong spatial correlation. The stronger the links are correlated, the lower the compression ratio. This is intuitive because when two links share a large amount of information, the shared information only needs to be recorded once, hence yielding a large compression savings. In Table 5, we find that the spatial correlation is very weak at the SNMP level (compression ratio is approx. 1), but much 


\begin{tabular}{|c|c|c|c|}
\hline Data Set & Entropy & Model & Empirical \\
\hline SNMP & $H$ (BB1-out $)$ & 20.6862 & $20.6700(\max \mathrm{mse}<0.1499)$ \\
\cline { 2 - 4 } & $H$ (BB2-out $)$ & 20.8895 & $20.8785(\max \mathrm{mse}<0.1654)$ \\
\cline { 2 - 4 } & $H(\mathrm{C} 1$-in $)$ & 21.0468 & $21.0329(\max \mathrm{mse}<0.1618)$ \\
\cline { 2 - 4 } & $H(\mathrm{C} 1$-in,BB1-out $)$ & 26.1517 & $26.1254(\max \mathrm{mse}<0.2717)$ \\
\cline { 2 - 4 } & $H(\mathrm{C} 1$-in,BB2-out $)$ & 26.1432 & $26.3078(\max \operatorname{mse}<0.2945)$ \\
\hline
\end{tabular}

Table 4: Comparision of SNMP data entropy between model and empirical calculations

\begin{tabular}{|c|c|c|}
\hline Data Set & Entropy (bits) & Joint Compression Ratio \\
\hline SNMP & C1-in 21.0468 & C1-in and BB1-out 1.0021 \\
\cline { 2 - 3 } & BB1-out 20.6862 & C1-in and BB2-out 0.9997 \\
\hline \multirow{2}{*}{ NetFlow } & C1-in 160.8071*697 & C1-in and BB1-out 0.8597 \\
\cline { 2 - 3 } & BB1-out 159.6124*1730 & C1-in and BB2-out 0.8782 \\
\hline Full Trace & C1-in 706.3773*697 & C1-in and BB1-out 0.8649 \\
\cline { 2 - 3 } & BB1-out 736.1722*1730 & C1-in and BB2-out 0.8702 \\
\hline
\end{tabular}

Table 5: Comparison of entropy calculations in information gain for different measurement granularity

stronger at both NetFlow and all packet monitoring levels (compression ratio is between 0.5 to 0.8702 ). This result suggests that there is no need to coordinate SNMP data gathering at different monitoring points, while coordinated collection and shared information at all monitoring points can yield significant savings in terms of storage for widely deployed NetFlow and all packet monitoring.

Table 5 also shows the information content comparison among the three monitoring options. SNMP data takes about 21 bits to encode per second, while NetFlow takes 74444bits per second, and all packet monitoring takes 492082 bits per second.

Now let's turn to the information gap between SNMP data and actual flow rate vector as studied in Section5.2.2. For the case under study, there are 4 links and 4 flow classes between 4 incoming-outgoing link pairs. The routing matrix is

$$
A=\left[\begin{array}{llll}
1 & 1 & 0 & 0 \\
0 & 0 & 1 & 1 \\
1 & 0 & 1 & 0 \\
0 & 1 & 0 & 1
\end{array}\right]
$$

Row vectors of $A$ are dependent:

$$
A_{4 .}=A_{1} .+A_{2 .}-A_{3 .},
$$

which means you can obtain the rate on link 4 as

$$
R_{4}=R_{1}+R_{2}-R_{3} .
$$

Therefore,

$$
\begin{aligned}
h\left(R_{I}\right) & =h\left(R_{1}, R_{2}, R_{3}\right) \\
& =\frac{1}{2} \log \left\{(2 \pi e)^{3}\left|A_{1-3,} \cdot K^{J} A_{1-3,}^{T} \cdot\right|\right\}
\end{aligned}
$$

We obtained the rate variance of each flow classes as:

$$
K^{J}=\left[\begin{array}{cccc}
1.21 & 0 & 0 & 0 \\
0 & 1.18 & 0 & 0 \\
0 & 0 & 0.239 & 0 \\
0 & 0 & 0 & 0.42
\end{array}\right] \times 10^{11}
$$

According to (29), the information content in the rate vector for all 4 flow classes is $h\left(R^{J}\right)=79.87$; according to (28), the information content in the rate vector for all 4 links is $h\left(R_{I}\right)=61.08$. The information gap is $23.53 \%$ of the total flow rate information. For this very simple topology and routing matrix, even if we collect SNMP data on all incoming and outgoing links, we still cannot fully infer the traffic rates between all incoming and outgoing pairs. We conjecture that as the topology and routing gets more complex, the information gap between the SNMP link rate vector and the flow rate vector increases, in other words, it becomes more difficult to obtain the traffic matrix by just looking at the SNMP data.

\section{Conclusion and Future Work}

Our goal in this paper was to put together a framework in which we could pose, and answer, challenging yet very practical questions faced by network researchers, designers, and operators, specifically i) how much information is included in various types of packet traces and by how much can we compress those traces, and ii) how much joint information is included in traces collected at different points and how can we take advantage of this joint information?

We obtained a number of interesting results. For example, we derived an information theoretic bound for the 
information content in traces collected at a single monitoring point and therefore were able to quantify the potential benefit of lossless compression on those traces. Not surprisingly, we found that the compression ratio (or information content) is small in SNMP traces, much higher in NetFLow traces, and extremely high in full packet traces. This shows that full packet capture does provide a quantum leap increase in information about network behavior. However, deploying full packet capture stations can be expensive. The interesting comparison, then, is that between the additional cost of full packet capture (say compared to NetFlow capture), and the additional amount of information produced by full packet traces (say compared to NetFlow traces). We are currently working on this problem, but early results seem to indicate that the increase in information content is proportionally larger than the increase in cost, suggesting that a full packet monitoring system gives you "more bang for the buck" (or rather "more entropy for the buck").

We also found that full packet header traces can be compressed in practice down to a minimum of $20 \%$ of their original size, and that the amount of compression is a function of the average flow size traversing that node: the larger the average flow size, the smaller the compression ratio.

In practice, packet traces are typically captured at multiple points. Therefore, it is important to understand how much information content is available in a set of traces, when that set is considered as a whole (as opposed to as a set of independent traces). This is turn is crucial to tackle further problems such as how many monitoring stations to set up (there might be a point of diminishing returns at which additional stations do not bring in enough "fresh" new information) and how to process and analyze those correlated data traces.

Using our framework, we find that joint coding or compression of traces further reduces the marginally compressed traces at the individual monitors. Specifically, the joint compression ratio (or equivalently, the additional compression benefit brought by joint coding of traces) is low for SNMP or byte/packet count traces, higher for NetFlow or flow-level traces, and significantly higher for packet-level traces. This means, for example, that joint coding would be very useful for full packet trace data (and to a lesser extent for NetFlow data) collected from different monitoring points and sent back to a NOC or central analysis station. We are now working on extending the work in this paper to joint coding techniques for large scale backbone and wireless networks.

\section{References}

[1] http://dag.cs.waikato.ac.nz/.
[2] http://www.cisco.com/warp/public/732/tech/netflow. Cisco NetFlow.

[3] Choi, B.-Y., And Bhattacharyya, S. On the accuracy and overhead of cisco sampled netflow. In Sigmetrics Workshop on Large Scale Network Inference (LSNI): Methods, Validation, and Applications (June 2005).

[4] Chou, J., Petrovic, D., And Ramchandran, K. A distributed and adaptive signal processing approach to reducing energy consumption in sensor networks. In IEEE Infocom 2003 (April 2003).

[5] Cover, T. A., And Thomas, J. A. Information theory. John Wiley \& Sons, Inc., 1991.

[6] Cristescu, R., Beferull-Lozano, B., And VETTERLI, M. On network correlated data gathering. In INFOCOM (Hong Kong, 2004).

[7] Duffield, N., And Grossglauser, M. Trajectory sampling with unreliable reporting. In IEEE Infocom (March 2004).

[8] Duffield, N., Lewis, J. T., O’Connell, N., Russell, R., AND ToOmey, F. Entropy of atm traffic streams: A tool for estimating qos parameters. IEEE JSAC, 13 (1995), 981-990.

[9] Duffield, N., Lund, C., And Thorup, M. Properties and prediction of flow statistics from sampled packet streams. In IMW'02: Proceedings of the 2nd ACM SIGCOMM Workshop on Internet measurment (New York, NY, USA, 2002), ACM Press, pp. 159-171.

[10] Fraleigh, C., Moon, S., Lyles, B., Cotton, C., Khan, M., Moll, D., Rockell, R., Seely, T., AND DiOT, C. Packet-level traffic measurements from the sprint IP backbone. IEEE Network (2003).

[11] Hohn, N., AND VeITCH, D. Inverting sampled traffic. In ACM/SIGCOMM Internet Measurement Conference (November 2003).

[12] Hohn, N., Veitch, D., AND Abry, P. Cluster process, a natural language for network traffic. In IEEE Transactions on Signal Processing, Special Issue on Signal Processing in Networking (August 2003), vol. 51, pp. 2229-2244.

[13] Hohn, N., Veitch, D., Papagiannaki, K., AND DIOT, C. Bridging router performance and queuing theory. In ACM Sigmetrics (New York, 2004). 
[14] Iannaccone, G., Diot, C., Graham, I., And McKeOwn, N. Monitoring very high speed links. In Proceedings of ACM Internet Measurement Workshop (November 2001).

[15] K. McCloghrie, M. T. R. Rfe 1213.

[16] LeE, W., AND Xiang, D. Information-theoretic measures for anomaly detection. In IEEE Symposium on Security and Privacy (2001).

[17] Leland, W., TAqQu, M., Willinger, W., AND WILSON, D. V. On the self-similar nature of ethernet traffic. IEEE/ACM Transactions on Networking 2, 1 (February 1994).

[18] LiU, X., AND SRIKant, R. An informationtheoretic view of connectivity in wireless sensor networks. In IEEE SECON (Conference on Sensor Networks and Adhoc Communications and Networks) (2004).

[19] LiU, Y., Towsley, D., Weng, J., AND GoeCKel, D. An information theoretic approach to network trace compression. Tech. Rep. UMASS CMPSCI 05-03, University of Massachusetts, Amherst, 2004.

[20] PAninski, L. Estimation of entropy and mutual information. Neural Computation 15, 1191-1254 (2003).

[21] Parulekar, M., And Makowski, A. $M / G / \infty$ input processes: A versatile class of models for network traffic. In Proceedings of INFOCOM (1997).

[22] Pattem, S., Krishnamachari, B., And Govindan, R. The impact of spatial correlation on routing with compression in wireless sensor networks. In IPSN'04: Proceedings of the third international symposium on Information processing in sensor networks (2004), pp. 28-35.

[23] Peuhkuri, M. A method to compress and anonymize packet traces. In Proceedings of ACM Internet Measurement Workshop (November 2001).

[24] Plotkin, N., And Roche, C. The entropy of cell streams as a traffic descriptor in atm networks. In IFIP Performance of Communication Networks (October 1995).

[25] Zhang, Y., Duffield, N., Paxson, V., And SHENKER, S. On the constancy of internet path properties. In Proc. ACM SIGCOMM Internet Measurement Workshop (2001).
[26] Zhang, Y., Roughan, M., Lund, C., And DONOHO, D. An information-theoretic approach to traffic matrix estimation. In ACM SIGCOMM (August 2003). 